\section{Multidisciplinary \\ SCIENTIFIC JOURNAL OF \\ MARITIME RESEARCH}

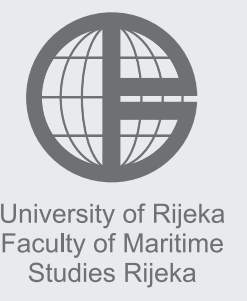

Multidisciplinarni

znanstveni časopis

POMORSTVO

\title{
Decentralized Agent-based Electronic Marketplace Supply Chain Ecosystem
}

\author{
Roman Gumzej ${ }^{1}$, Dragan Čišić ${ }^{2}$ \\ ${ }^{1}$ University of Maribor, Faculty of logistics, Mariborska cesta 7, Celje, Slovenia, e-mail: roman.gumzej@um.si \\ ${ }^{2}$ University of Rijeka, Faculty of Maritime Studies, Studentska 2, 51000 Rijeka, Croatia, e-mail: dragan@pfri.hr
}

\begin{abstract}
Electronic marketplaces have become an essential part of the business to business e-commerce. Supply chain concept is fully taking advantage of using the network effects in electronic marketplaces due to augmented added value from each participant. However, highly desirable network effects lead to one company monopoly over the whole marketplace. To prevent this effect, authors are proposing and devising a decentralized agent-based electronic marketplace for supply chain ecosystem. The goal of our research is to improve overall supply chain service quality by allowing companies' agents to evaluate the service quality of their partners through the history of their transactions. Consequently, since in their procurement activities more informed decisions are being made instantaneously, continuously and autonomously at each node of a supply chain, supply chain service quality is being improved along the whole supply chain.
\end{abstract}

\section{ARTICLE IN FO}

Preliminary communication

Received 19 March 2018

Accepted 8 May 2018

\section{Key words:}

E-marketplace

Agent-based system

Supply chain management

Ontology

Platform

\section{Introduction}

Companies have nowadays realized that if they want to prosper in the intensely competitive world, it is necessary to organize themselves and develop strategies to utilize the opportunities of the supply chain networks, and especially network effects that are emerging from and mutually supported by their customers and suppliers. In other words, current marketplaces are guided not by individual companies, but rather by a constellation of connected business entities that are pursuing competitive advantage by competing and occasionally cooperating with other entities.

With increasing competition in the global market, organizations are beginning to realize that it is not enough to merely improve their efficiency internally, but it is also necessary to consider the competitiveness and efficiency of their supply chains. Performance measurement and optimization play a vital role in improving the competitiveness of supply chains [1]. Moreover, with the rapidly developing world economy and global marketplaces, there has been a drastic increase in the pressure on organizations to find new ways to create and deliver values to customers through supply chain management. There has been a growing recognition of the importance of building relationships with customers for improvements in profitability, as well as serviceability and reduced costs throughout the supply chain [2].

E-marketplaces have become an essential part of e-commerce. The centralized web-site based approach is being successfully exploited by companies not being connected by long-term contracts and direct e-business relations. Multiple e-marketplaces of such type have been established, mostly being used by companies of a common industry, to help them manage their supply chains.

Weyl in [3] has shown that over time, network effect produces a monopoly in which single company controls the entire marketplace. This has not yet happened in supply chain marketplaces, although the current situation in dissimilar ecosystems is supporting this fact (Uber and taxi marketplaces for example). Indications of a similar occurrence are currently appearing in the maritime transport ecosystem, where digital cooperation of Alibaba OneTouch, CMA CGM [4] and Maersk [5] is endangering the operation of freight forwarders and shipping agents. OneTouch system has all the prerequisites to create a monopoly in the marketplace. 
In order to prevent the single company to control the marketplace, a decentralized agent-based electronic marketplace for supply chain ecosystem is presented.

In the framework of our research, a decentralized agent-based e-marketplace platform has been devised. When companies arise, they are assigned to agents that represent them in the marketplace and are registered with a yellow-page service corresponding with their line of business in general and their products in particular. While companies arise and vanish they are registered and de-registered from this service. This service, being used by all companies' agents on the e-marketplace, manages the routing of their inquiries for specific goods. Once the proposals have arrived from supplier companies' agents in reply to a customer's inquiry, a selection and ordering procedure is conducted between a customer and his supplier agents until the transaction is completed.

In this article, an e-marketplace ontology for supply chain automation is presented. First, contemporary e-marketplaces are reviewed. Then the proposed multiagent e-marketplace framework is presented - its knowledge sharing, behavioral and communication parts. As a conclusion, the expected impact on using our framework in supply chain automation is discussed.

\section{A Motivation}

Many authors [3] emphasize the importance of delivering superior total value to the customer in terms of promptness, cost, quality, and flexibility rather than by focusing solely on promptness and cost. According to [4] service quality has been a major area of attraction for practitioners and researchers. Its proven relationship together with improving business performance by lowering costs, increasing customer satisfaction, achieving customer loyalty and increasing profitability has further motivated both researchers and practitioners to explore this area.

The greatest problem that modern organizations face today is their inability to quickly adapt themselves to changes dictated by fast-evolving supply chains. More specifically, organizations currently rely on various mechanisms to perform quality assessment of their partners and adapt their decision-making processes to improve their business performance. Hence, numerous research studies have dealt with the subject of service quality in supply chains and a consensus has been reached concerning a strong relationship between service quality and supply chain performance [5].

To achieve the desired global optimization throughout the entire supply chain, one must employ proper mechanisms to measure, monitor and control service quality through an interorganisational assessment system [6]. Such systems are sensibly implemented to identify opportunities for improved supply chain efficiency and competitiveness, to help understand how companies, operating in supply chains, affect each other's performance, to support the supply chain in satisfying consumer requirements and to assess the result of an implemented initiative [7]. As stated by [8] for collaborative networks, supply chains also have the need for decentralized operations where partners conduct their planning autonomously and only exchange a limited amount of information. A frequently proposed solution concept for this purpose is auction mechanisms and, more specifically, multi-agent systems.

Decentralization changes paradigms of established electronic marketplaces in which the corporation that controls the platform controls all aspects of the exchange of information, goods, services, and payments. Agentbased marketplace leverages agent technology to perform all marketplace functions in a decentralized way with increased efficiency, resulting in reduced costs and increased trust among participants.

\section{B Methods}

According to [11] automation of supply chain management systems has long been a principal concern of both academia and industry. Generally, two approaches of service transformation in the digital era can be observed for efficient, service-quality-oriented supply chain management:

(1) Centralised e-marketplace web portals, combining demand and supply in a temporally and spatially shared repository $[12,13]$ and

(2) Decentralised, agent-based e-marketplaces with a yellow-page dictionary service and distributed network of temporally and spatially distributed agents of supply chain partners [11,14-25].

Each approach focuses on enriching some aspects of traditional supply chain management information systems. The centralized e-marketplace approach facilitates interoperability amongst supply chain participants, regardless of their information system diversity. On the other hand, the participants are left with a limited degree of autonomy, and without the capacity to adapt in a dynamic way to changing real-world situations. In case such situations arise, intelligent agents can provide the participating systems with a high degree of autonomy and dynamism [11].

\section{Goal}

The goal of this research is to improve overall supply chain service quality by allowing companies' agents to evaluate the service quality of their partners through the history of their transactions. Any transaction in progress is automatically subjected to a service quality-oriented decision support system [9], where the current transaction data, supplemented with historical data on previous transactions with the prospective suppliers, are evaluated with the goal of best meeting the customers' needs. Consequently, since more informed decisions are taking place continuously and autonomously, supply chain service quality is being improved along the whole supply chain. 
In contrast to centralized e-marketplace platforms, the agent-based approach is distributed and dynamic, and hence corresponds to the supply chains' natural behavior. It relies on the holistic approach to service quality assessment defined in $[9,10]$. The main reasons for employing a holistic approach are, in consequence of the otherwise identified absence of connection with a strategy, lack of system thinking, in which a supply chain must be viewed as a whole entity, lack of balanced approach integrating financial and non-financial measures, as well as loss of supply chain context, thus encouraging local optimisation.

Based on the service quality assessment model, a novel multi-agent based collaboration framework for interorganisational cooperation and service quality assessment is introduced that can perform supply chain management operations autonomously, according to the mentioned goals.

In the sequel, the proposed multi-agent based collaboration framework with interorganisational (supply chain) service quality assessment is presented.

\section{Multi-Agent Framework for Supply Chain Automation}

According to [27] agent architectures are fundamental mechanisms underlying the autonomous components that support effective behavior in real-world, dynamic and open environments.

In artificial intelligence, an intelligent agent is an autonomous entity which observes through sensors and acts upon an environment using actuators (i.e. it is an agent) and directs its activity towards achieving goals (i.e. it is rational), as defined in economics [28]. Intelligent agents may also learn or use knowledge to achieve their goals.

To apply the decentralized agent-based approach to supply chain management, any pair of supply chain nodes shall be autonomously integrated into both-the information and material flow-with the goal of meeting supply chain nodes' service quality requirements. By their behavior, they are classified as model-based learning agents.

\section{A Knowledge sharing}

Each multi-agent system needs a mechanism for knowledge sharing. Agent communication protocols may be utilized to access this knowledge and to establish sensible relations among supply chain nodes as well as to implement appropriate behavioral models.

According to [29] ontologies were developed in artificial intelligence (AI) to facilitate knowledge sharing and reuse. They enable a shared and common understanding of a defined domain that can be communicated between people and application systems. In our case, it is meant for conceptualizing and managing supply chain knowledge, agent communication protocols by which this knowledge is shared between supply chain nodes and autonomous agent behavior models.
Our ontology establishes the basic vocabulary of terms which are relevant for e-commerce. Hence, we have named it e-marketplace (see Fig. 2). The main concepts it introduces are associated with supply chain nodes before and after the transformation, according to our ontology (see Fig. 1a and 1b), represented by agents and transactions that are exchanged among them. It was created using the Stanford's Protégé tool.

Class Transaction represents business transactions among individual nodes of our supply chain. These business transactions are assigned to class Agent, which can

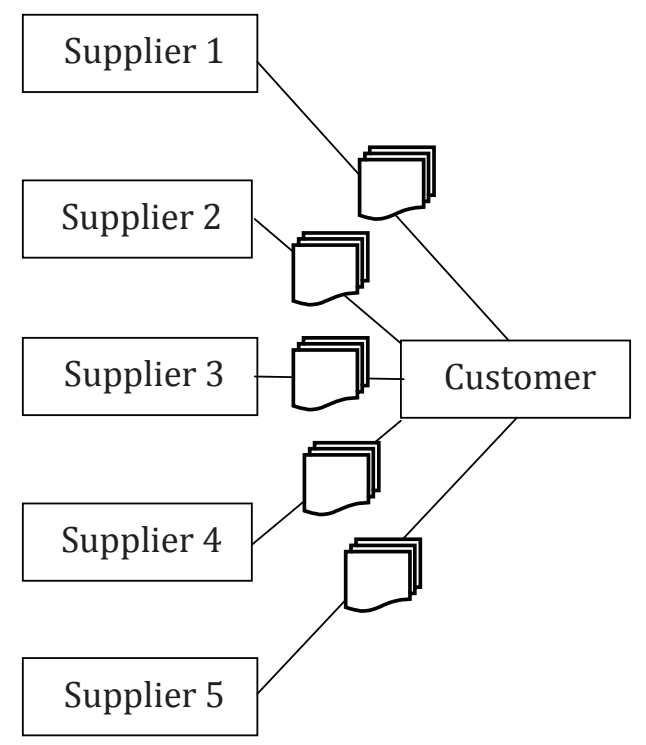

Figure 1a Supply chain example (before transformation) (Transactions in various forms and protocols (EDI, e-mail, Fax,...)

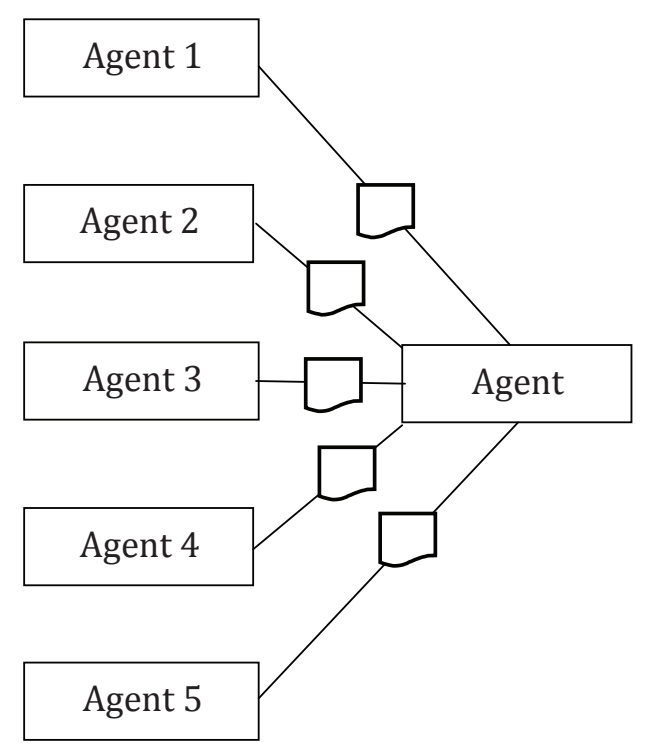

Figure 1b Supply chain example (after transformation) (Transactions in the form and protocol, determined by the ontology)

Figure 1 Supply chains before and after admission in the platform 


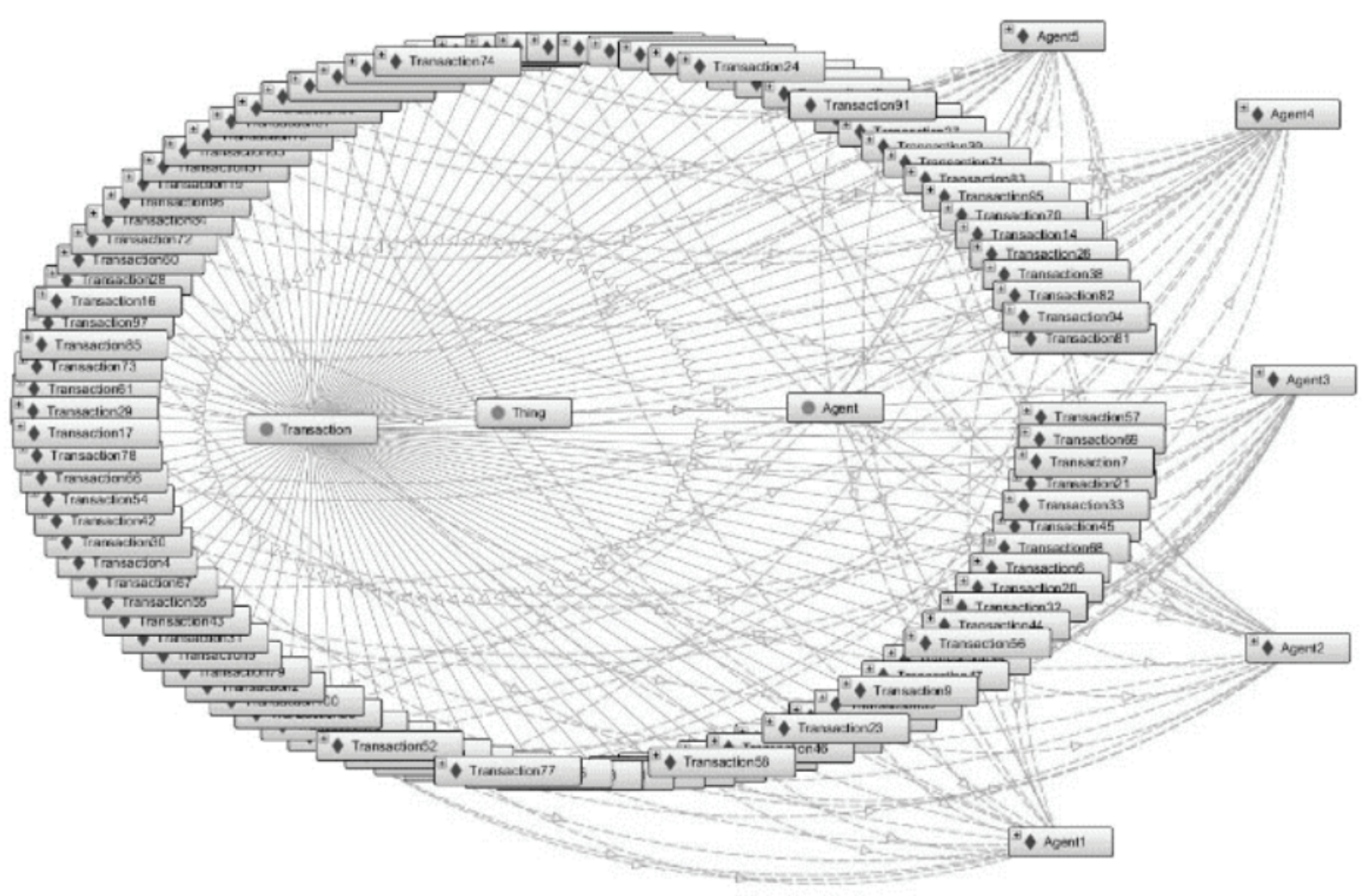

Figure 2 E-marketplace ontology example

represent different types of supply chain nodes. Classes Transaction and Agent are disjoint, which means that any individual node (object) cannot be an instance of more than one of these two classes. In our example (see Fig. 2) we have defined 100 transactions and split transaction objects evenly among our 5 supplier agent objects (Agent 1 to 5), who are members of our customer agent's (Agent) supply chain.

In the sequel agent communication protocols, which are needed to perform supply chain operations and utilize knowledge shared by the e-marketplace ontology, are presented.

\section{B Communication}

A key component in any multi-agent based system is communication. According to [27], agents need to be able to communicate with users, system resources and with each other to collaborate and negotiate. In particular, agents interact with each other by using agent communication language (ACL).

According to FIPA (the Foundation for Intelligent, Physical Agents), each agent has its own characterization, which represents its behavior. Agents' messages represent actions or communicative acts, also known as speech acts of performatives. It is stated in the FIPA standards that, to be fully compliant, agents must be able to receive any legal FIPA-ACL message and at the very least respond with a not-understood message, in case the processing of the message does not render a meaningful result. FIPA-ACL messages contain a set of one or more message parameters. The selection of parameters, which are needed for effective agent communication, varies according to the situation [27].

Our autonomous agents communicate over HTTP protocol and are based on the FIPA Contract Net Protocol [30], which allows one agent, the Initiator, to have some tasks performed by one or more other agents (Participants) and further optimization of a function that characterizes the task. For a given task, any number of Participants may respond with a proposal; the rest must refuse. The Initiator then continues negotiations with the participants that proposed.

In Fig. 3 the communication among the five agents, representing suppliers, and the one agent representing a customer is shown, according to the FIPA Contract Net Protocol for our e-commerce ontology. First, the customer object reads the data on the potential supplier objects. Then it contacts them with a call for proposal (CFP) message indicating transaction data. The supplier objects reply with their proposals (PROPOSE) according to their transaction data. After comparing the incoming proposals and selecting the most appropriate one, the customer object informs the owner of the selected transaction that it accepts its proposal (ACCEPT-PROPOSAL) and the rest that it rejects their proposals (REJECT-PROPOSAL). The 


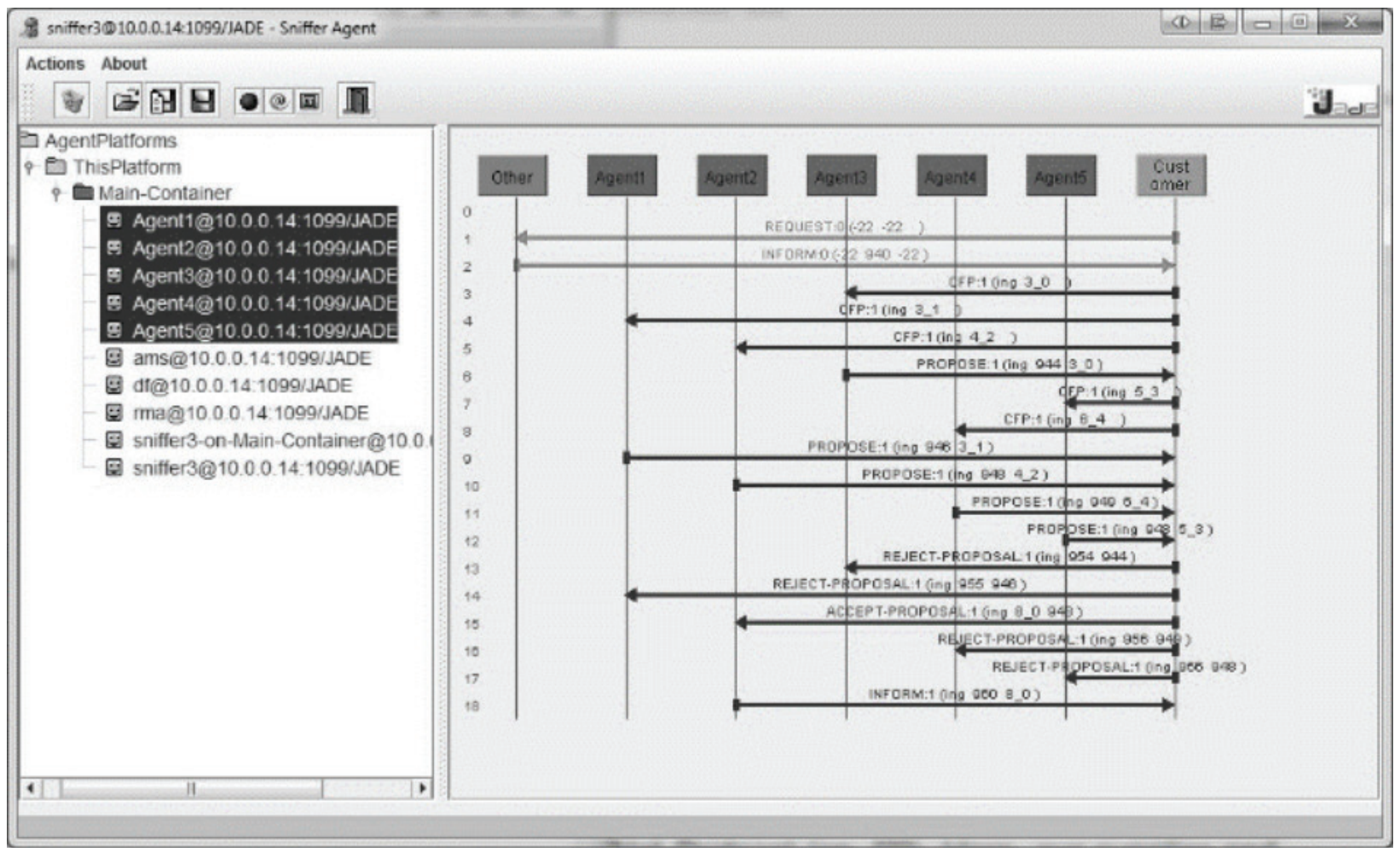

Figure 3 E-marketplace communication protocol example scenario

selected supplier object fulfills the order of the customer object by informing it about this fact, which results in appropriate fulfillment actions.

\section{Behavior}

All agents are generic until they are enlisted as members of a supply chain. When agents are registered in yellow page service, their specific role (customer, supplier, customer, and supplier) is indicated. Every pair of supply chain nodes that interact with each other can be observed as a customer-supplier relation. A customer orders a material, service or finished product (item) from its suppliers based on their respective price and service quality.

When an agent of a type supplier is being registered in yellow page services, the query is made to the e-marketplace ontology for that agent. Based on this query, agentrelated indicators are saved into a SupplierContentMessage object, which consists of the following information:

- Correctness: average value of the Correctness indicator for all business transactions made in its history of participating in the supply chain;

- Timeliness: average value of the Timeliness indicator for all business transactions made in its history of participating in the supply chain;

- Dependability: average value of the Dependability indicator for all business transactions made in its history of participating in the supply chain;
- Offered price: the value of the current offered price for a specific item;

- Agent name: unique identifier of the supplier.

Analogously, when an agent of a type customer is being registered in yellow page services, the query to the e-marketplace ontology is made for all eligible suppliers in the supply chain. Based on this query, customer-related indicators are saved into a CustomerKnowledge object, which consists of the following information:

- Agent name: unique identifier of the customer;

- Maximum price: maximum price from all customer suppliers' business transactions.

In our order-cycle, the supplier and customer nodes take over the initiator and participant roles respectively, according to the FIPA Contract Net Protocol. The transaction data is transferred between them with appropriately tagged messages.

When a specific proposal is made by a supplier, a serialized object SupplierContentMessage is contained in a suppliers' message content. Based on that known suppliers' message content (average values of the indicators) and the message content about a specific supplier (stored in the CustomerKnowledge object) that is already known to the customer (offered price and maximum price), the overall price indicator is calculated. As all indicators are now known to a customer, a service quality of each supplier may be calculated next. 
The service quality for any supply chain node is maintained by its agent within its knowledge base. Initially (since there are no previous service quality data), the decision on an order is made solely based on price. With any subsequent order, being fulfilled, however, the service quality is recalculated and the proposal selection decisions are made in an increasingly informed manner.

Let us consider service quality assessment in a typical ordering operational cycle (cf. Fig. 3):

(1) A call for proposals (CFP) for an item is posted to all known suppliers; it contains the number of items we wish to order and the requested date of order fulfillment.

(2) Suppliers either PROPOSE their offers and provide the data on the price for the requested amount of items or REFUSE the request.

(3) The customer makes the decision on the acceptance of a particular offer based on the prices and the service quality indicators of all suppliers that provided positive feedback to our inquiry.

(4) An order is made to the selected supplier by posting it an ACCEPT-PROPOSAL, while a REJECT-PROPOSAL is sent to all other participating suppliers.

(5) The order is either acknowledged as being fulfilled or a failure to fulfill the order is issued by the chosen supplier.

(6) Based on the outcome of the inquiry, service quality indicators of the supplier-nodes are recalculated to be considered in the next ordering operation.

The communication between different types of agents starts when a customer requests a quotation (call for proposal) for his desired item. After calls for proposals are made, proposals are sent from eligible registered suppliers to the customer. Based on the own knowledge about suppliers and indicators sent from suppliers, the service quality calculation is done and a decision is made accordingly. If a procurement decision for a supplier's proposal is positive, the supplier is notified about proposal acceptance, otherwise, a proposal refusal is sent to the supplier. Finally, the current transaction is saved into the e-marketplace ontology of the supplier to be accounted for in its future proposal considerations

\section{Conclusion}

Today, companies have realized that, in order to survive in the contemporary business environment, they need to incorporate the opportunities of the supply chain marketplace ecosystem. Due to the fact that the network effect leads to a monopoly in marketplaces, the design of a decentralized agent-based e-marketplace has been presented. This approach has been adopted, since it resembles to the natural behavior of supply chain nodes, which appear, register their services, handle inquiries and orders and establish their own service quality-based supply chains on the supply chain network with their partners.
In this paper, the knowledge sharing, behavior, and communication models of the proposed multi-agent framework with a service-quality oriented assessment model for collaboration with supply chain partners have been presented. The method for assessing their service quality and its adoption into supply chain management operations has been presented.

Considering the goal of our research, which was to improve overall supply chain service quality by allowing companies' agents to evaluate the service quality of their partners through the history of their transactions, we may consider it achieved by the proposed agent-based e-marketplace framework. Since any echelon in a supply chain can be characterized as a supplier, a customer or a combination of both and the fact that more informed decisions are taking place continuously and autonomously with every transaction, we may generalize the result of our experiment and state that by our approach supply chain service quality is being improved along the whole supply chain.

With the advent and deployment of the Blockchain technology, its benefits are also being introduced into decentralized electronic marketplaces, providing for their strengthened confidentiality, integrity, and availability [32].

Finally, decentralization has the power to complement, challenge and even exceed functions of traditional established electronic marketplaces.

\section{References}

[1] W. Zhang and M. Reimann, "Towards a multi-objective performance assessment and optimization model of a two-echelon supply chain using SCOR metrics," Central European Journal of Operations Research, 22(4): pp. 591-622, 2014.

[2] R. Niraj, M. Gupta, and C. Narasimhan, "Customer profitability in a supply chain. Journal of Marketing," 65(3): pp. 1-16, 2001.

[3] Weyl.EG A price theory of multisided platforms The American Economic Review, Vol. 100, No. 4, pp. 1642-1672.

[4] Signature of a digital cooperation between CMA CGM and Alibaba OneTouch, https://www.cma-cgm.com/news/1496/ signature-of-a-digital-cooperation-between-cma-cgm-andalibaba-onetouch accessed on March 2018.

[5] Alibaba Is Teaming Up with This Shipping Giant http://fortune.com/2017/01/04/alibaba-maersk-shipping-partnership-onetouch/ accessed on March 2018.

[6] D.J. Ketchen et al., "Best value supply chains: A key competitive weapon for the 21st century," Business Horizons, 51(3): pp. 235-243, 2008.

[7] N. Seth, S. Deshmukh, and P. Vrat, "A framework for measurement of quality of service in supply chains," Supply Chain Management: An International Journal, 11(1): pp. 8294, 2006.

[8] G. Chow, T.D. Heaver, and L.E. Henriksson, "Logistics performance: definition and measurement," International Journal of physical distribution \& logistics management, 24(1): p. 17-28, 1994.

[9] B. Sahay et al., "A conceptual model for quality of service in the supply chain," International Journal of Physical Distribution \& Logistics Management, 36(7): pp. 547-575, 2006. 
[10] A. Lyons et al., Supply Chain Performance Measurement, in Customer-Driven Supply Chains, Springer London, pp. 133148, 2012.

[11] M. Mes, M. van der Heijden, and P. Schuur, "Interaction between intelligent agent strategies for real-time transportation planning," Central European Journal of Operations Research, 21(2): pp. 337-358, 2013.

[12] R. Gumzej and B. Gajšek, Introducing quality of service criteria into supply chain management for excellence, Technological Solutions for Modern Logistics and Supply Chain Management, p. 70-, 2013.

[13] R. Gumzej, P. Sukjit, and H. Unger, "Modelling Overlay Networks for Autonomous Supply Chain Systems," Logistics \& Sustainable Transport, 3(2), 2012.

[14] K. Pal and B. Karakostas, "A multi agent-based service framework for supply chain management," Procedia Computer Science, 32: pp. 53-60, 2014.

[15] W. Rashad and R. Gumzej, "The Information Technology in Supply Chain Integration: Case Study of Reda Chemicals with Elemica," International Journal of Supply Chain Management, 3(1), 2014.

[16] K. Kim, B.C. Paulson Jr, and C.J. Petrie Jr., "Agent based electronic markets for project supply chain coordination", Proceedings of the AAAI-00 Workshop on Knowledge-Based Electronic Markets, USA, 1999.

[17] Y. Kovalchuk, A Multi-agent decision support system for supply chain management, 2009 [Citeseer].

[18] H.A. Rady, "Multi-agent system for negotiation in a collaborative supply chain management," International Journal of Video \& Image Processing and Network Security IJVIPNSIJENS, 11(5), 2011.

[19] L.A. Bearzotti, E. Salomone, and O.J. Chiotti, "An autonomous multi-agent approach to supply chain event management," International Journal of Production Economics, 135(1): pp. 468-478, 2012.

[20] F. Ameri and C. McArthur, "A multi-agent system for autonomous supply chain configuration," The International Journal of Advanced Manufacturing Technology, 66(5-8): pp. 10971112, 2013.

[21] R. Domínguez, S. Cannella, and J.M. Framinan. "SCOPE: A Multi-Agent system tool for supply chain network analysis,"
EUROCON 2015-International Conference on Computer as a Tool (EUROCON), IEEE, IEEE, 2015.

[22] R. Dominguez et al. "Using multi-agent systems to explore information sharing in arborescent supply chain networks," Industrial Engineering and Systems Management (IESM), Proceedings of 2013 International Conference on, IEEE, 2013.

[23] G. Wang, T. Wong, and X. Wang, "An ontology based approach to organize multi-agent assisted supply chain negotiations," Computers \& Industrial Engineering, 65(1): pp. 2-15, 2013.

[24] J.E. Hernández et al., "Collaborative planning in multi-tier supply chains supported by a negotiation-based mechanism and multi-agent system," Group Decision and Negotiation, 23(2): pp. 235-269, 2014.

[25] P. Sitek, I.E. Nielsen, and J. Wikarek, "A hybrid multi-agent approach to the solving supply chain problems," Procedia Computer Science, 35: pp. 1557-1566, 2014.

[26] J. Fu and Y. Fu, "An adaptive multi-agent system for cost collaborative management in supply chains," Engineering Applications of Artificial Intelligence, 44: pp. 91-100, 2015.

[27] S. Kumari et al., "A multi-agent architecture for outsourcing SMEs manufacturing supply chain," Robotics and Computer-Integrated Manufacturing, 36: pp. 36-44, 2015.

[28] M. Giannakis and M. Louis, "A multi-agent based framework for supply chain risk management," Journal of Purchasing and Supply Management, 17(1): pp. 23-31, 2011.

[29] F.L. Bellifemine, G. Caire, and D. Greenwood, Developing multi-agent systems with JADE. Vol. 7, John Wiley \& Sons, 2007.

[30] S. Russell, and P. Norvig. Artificial Intelligence: A modern approach. Prentice-Hall, Englewood Cliffs, 25: p. 27-, 1995.

[31] D. Fensel, “Ontologies," Ontologies, Springer Berlin Heidelberg. pp. 11-18, 2001.

[32] F. Bellifemine, A. Poggi, and G. Rimassa, "Developing multiagent systems with a FIPA-compliant agent framework," Software-Practice and Experience, 31(2): pp. 103-128, 2001.

[33] H. Subramanian, "Decentralized Blockchain-based Electronic Marketplaces”, Communications of the ACM, 61(1): pp. 78-84. 